\title{
An Integrated Circuit for Chip-Based Analysis of Enzyme Kinetics and Metabolite Quantification
}

\author{
Boon Chong Cheah, Alasdair I. Macdonald, Christopher Martin, Angelos J. Streklas, Gordon \\ Campbell, Mohammed A. Al-Rawhani, Balazs Nemeth, James P. Grant, Michael P. Barrett, and David \\ R. S. Cumming, Fellow, IEEE
}

\begin{abstract}
We have created a novel chip-based diagnostic tools based upon quantification of metabolites using enzymes specific for their chemical conversion. Using this device we show for the first time that a solid-state circuit can be used to measure enzyme kinetics and calculate the Michaelis-Menten constant. Substrate concentration dependency of enzyme reaction rates is central to this aim. Ion-sensitive field effect transistors (ISFET) are excellent transducers for biosensing applications that are reliant upon enzyme assays, especially since they can be fabricated using mainstream microelectronics technology to ensure low unit cost, mass-manufacture, scaling to make many sensors and straightforward miniaturisation for use in point-of-care devices. Here, we describe an integrated ISFET array comprising $\mathbf{2}^{16}$ sensors. The device was fabricated with a complementary metal oxide semiconductor (CMOS) process. Unlike traditional CMOS ISFET sensors that use the $\mathrm{Si}_{3} \mathrm{~N}_{4}$ passivation of the foundry for ion detection, the device reported here was processed with a layer of $\mathrm{Ta}_{2} \mathrm{O}_{5}$ that increased the detection sensitivity to $45 \mathrm{mV} / \mathrm{pH}$ unit at the sensor readout. The drift was reduced to $0.8 \mathrm{mV} / \mathrm{hour}$ with a linear pH response between $\mathrm{pH} 2$ - 12. A high-speed instrumentation system capable of acquiring nearly 500 fps was developed to stream out the data. The device was then used to measure glucose concentration through the activity of hexokinase in the range of $0.05 \mathrm{mM}-231 \mathrm{mM}$, encompassing glucose's physiological range in blood. Localised and temporal enzyme kinetics of hexokinase was studied in detail. These results present a roadmap towards a viable personal metabolome machine.
\end{abstract}

This work is supported by the Engineering and Physical Sciences Research Council grant number EP/K021966/1. BCC has a University of Glasgow Lord Kelvin Adam Smith Sensors Ph.D. studentship. MPB is part of Wellcome Trust Centre for Molecular Parasitology funded by the Wellcome Trust. BCC and AIM are joint first authors.

B. C. Cheah, C. Martin, A. J. Streklas, M. Al-Rawhani, J. Grant, and D. R. S. Cumming are with the Microsystems Technology Group, School of Engineering, University of Glasgow, Glasgow, G12 8LT, U.K. (e-mail: b.cheah.1@ research.gla.ac.uk; a.streklas.1@ research.gla.ac.uk; christopher.martin@glasgow.ac.uk; mohammed.al-rawhani@glasgow.ac.uk; james.grant@glasgow.ac.uk; david.cumming.2@glasgow.ac.uk).

B. Nemeth, was with the Microsystems Technology Group, School of Engineering, University of Glasgow, Glasgow, G12 8LT, U.K. He is now with the IMMS GmbH, Erfurt, Germany. (e-mail: balazs.nemeth@imms.de).

A. I. Macdonald, G. Campbell, and M. P. Barrett are in the Wellcome Trust Centre for Molecular Parasitology within the Institute of Infection, Immunity and Inflammation, University of Glasgow, Glasgow, G12 8TA U.K. M.P. Barrett is also at Glasgow Polyomics, University of Glasgow. (email: alasdair.macdonald@glasgow.ac.uk; gordon.campbell@glasgow.ac.uk; michael.barrett@glasgow.ac.uk).
Index Terms-Biosensor, CMOS, electrochemical sensor, enzyme kinetics, hexokinase, ion-sensitive field effect transistors (ISFET), metabolomics, point-of-care (POC) diagnostics.

\section{INTRODUCTION}

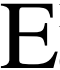
NZYMES are natural catalysts that accelerate the rate of chemical reactions in order to sustain life [1]-[6]. Since their discovery, enzymes have been increasingly exploited for commercial purposes such as in foods [7], detergents [8], and healthcare [9]. Most enzymes catalyse reactions following kinetics that are described by the Michaelis-Menten equation [1]-[6]. The rate of an enzyme's activity depends on its substrate concentration, hence measurements of enzyme activity can be used to determine a concentration of its substrate. The $K_{M}$ value is the substrate concentration ([S]) at which the reaction rate is half the maximum velocity $\left(v_{\max }\right)$ of the reaction [1]-[4].

$$
v=v_{\max } \frac{[S]}{[S]+K_{M}}
$$

By understanding the $K_{M}$ of enzymes, the rate and sensitivity of a wide range of biosensing and diagnostic devices can be greatly improved, including potential application in metabolomics.

Many enzymes consume or produce protons as part of their reaction pathway. It is therefore possible to perform enzyme assays using an electrochemical sensor such as an IonSensitive Field Effect Transistor (ISFET). The ISFET was first proposed by Bergveld [10], and the first practical application was to measure a penicillin [11]. The ISFET, a potentiometric device [12], measures the hydrogen concentration dependent surface potential when exposed to an aqueous solution, with respect to a reference electrode [13]. The earliest devices were made using specialist one-off processes, but more recently research has shown that good devices can be made using standard complementary metal oxide semiconductor processes [14]. Further work led to the implementation of arrays of sensors, much like a camera using photodiodes [15], [16]. Using these devices it has been possible to show that chemical imaging using sensor signal variation as the image contrast mechanism is possible. 
The array format also presents possibilities for improving overall system performance, which we exploit in this paper. As will be shown, if instead of one single sensor channel, we use multiple independent sensor channels with uncorrelated noise in each. Averaging over more than one sensor leads to a significant improvement in noise, hence effective sensitivity. To thoroughly explore this possibility a very large array of $2^{16}$ sensors has been deployed.

All ISFETs rely on a so-called dielectric membrane (an insulator). In solution a charge double-layer is formed as hydrogen or hydroxyl ion species, according to $\mathrm{pH}$, adsorb on to the surface forming a static charge layer. Charge equilibrium across the dielectric to the nearest floating gate electrode produces a localised and detectable signal on each sensor in an array. The sensitivity varies depending on the exact dielectric used. Several materials have been studied, including $\mathrm{SiO}_{2}, \mathrm{Si}_{3} \mathrm{~N}_{4}, \mathrm{Al}_{2} \mathrm{O}_{3}, \mathrm{SnO}_{2}, \mathrm{ZrO}_{2}$, and $\mathrm{Ta}_{2} \mathrm{O}_{5}$ [17][21]. $\mathrm{Ta}_{2} \mathrm{O}_{5}$ has proven to be an excellent material with good immunity against interference ions and a wide working $\mathrm{pH}$ range [22].

Using only a single ISFET sensor, various enzymes such as urease [22], [23], creatinine deiminase [24], and glucose oxidase [25] have been used to modify the surface of the ISFET gate to be sensitive towards a specific substrate. Nevertheless, enzyme kinetics (i.e. the change in velocity of the reaction catalysed by the enzyme as a function of substrate concentration) has not previously been studied using an ISFET-based sensor.

Three different enzymes have been widely used for glucose measurements: hexokinase, glucose oxidase (GOx) and glucose-1-dehydrogenase (GDH) [9]. Commercial glucose biosensors use GOx or GDH as the bioreceptor for the rapid analysis of blood glucose levels, informing the patient of his or her glycaemic status [9], [26]. Hexokinase is the first enzyme in glycolysis used to convert glucose into energy in the form of adenosine triphosphate (ATP) [27], and has been extensively studied in clinical laboratories using spectrophotometry as a reference method for glucose measurements [9].

In order to create a new and quantifiable measurement system for enzyme assays based on ISFETs, we have developed a new CMOS sensing array to provide speed and multiple data from which data can be accurately determined. We describe the kinetic analysis of hexokinase on the CMOS ISFET array and also a new approach using this enzyme for direct glucose measurements. The chip, which was fabricated in the AMS $0.35 \mu \mathrm{m}$ four metal CMOS process, consists of an array of $256 \times 256$ pixels. These pixels were subsequently post-processed with a $\mathrm{Ta}_{2} \mathrm{O}_{5}$ layer to improve the longevity, reduce drift, and increase the sensitivity of the sensor. Spectrophotometric assays were initially used to determine the activity of hexokinase supplied in solution to the chip. The response of hexokinase to its substrate (glucose) at various concentrations caused a voltage change corresponding to the production of hydrogen ions from the reaction, validating the assay as a means of direct glucose measurement. Using the measured data, the Michaelis-Menten constant $\left(K_{M}\right)$ of hexokinase on the chip was calculated using the maximum $\mathrm{d} V / \mathrm{d} t$, where $V$ is the ISFET signal voltage. Using the array function of the chip, we have explored the effect of averaging over different numbers of sensors, and its impact on the noise performance of the system. Hexokinase was thus validated as an enzyme capable of being used to determine glucose levels on ISFETs.

The work in this paper will promote future research towards the implementation of multiple assays on a single chip format. Such a device has the potential to produce personalised metabalomic measurements that are now becoming central to the development of precision and stratified medicine [28], [29].

\section{MATERIALS AND METHODS}

\section{A. $256 \times 256$ ISFET Array Chip}

The ISFET sensor array chip was fabricated in a commercial foundry (Austriamicrosystems, Austria) using the standard $0.35 \mu \mathrm{m}$, four metal process. As shown in Fig. 1a, each pixel comprises 4 transistors, where P2 is a PMOS ISFET transistor configured as a voltage-follower for hydrogen ion concentration detection, $\mathrm{P} 1$ is an in-pixel current source for $\mathrm{P} 2$, and $\mathrm{P} 3$ and $\mathrm{N} 1$ form a transmission gate for addressing using $\mathrm{V}_{\text {col }}$ and $\overline{\mathrm{V}_{\text {col }}}$ [16]. The size of each pixel is $10.2 \mu \mathrm{m} \times$ $10.2 \mu \mathrm{m}$, so that the entire array occupies an area of approximately $2.87 \mathrm{~mm} \times 2.87 \mathrm{~mm}$. An atomic force micrograph of a single pixel and an optical micrograph of the whole array are also shown in Fig. 1. The corrugated surface profile that can be seen in Fig. 1b arises from the top-metal electrode layout. Our devices show variation in the sensor threshold voltage with a standard deviation of $0.238 \mathrm{~V}$. However we study time response measurements where static offsets are eliminated.

Fig. 2 illustrates the architecture for reading all $256 \times 256$ pixels from the chip. The sensor array is divided into two halves of 256 rows and 128 columns. An identical but separate row multiplexing system and column decoder is used for each half. The 7-bit column decoders select one column from the 128. The row data is broken down into 4 pairs of parallel blocks of 32 analog signals at each side. The blocks are readout sequentially via a 1 from 8 multiplexer.

\section{B. Post-Processing of the CMOS Chip}

The cross-section shown in schematic form of a pixel, illustrating the semiconductor, metal and dielectric layers, is shown in Fig. 3. The metal layout in each pixel uses a metalvia stack to contact the underlying transistor to the top metal layer (MET 4) beneath the dielectric passivation layer. The MET 4 plate therefore forms a capacitor with the analyte above the chip [16]. The dielectric in contact with MET 4 acts as an ion sensing material which translates the hydrogen ion concentration in the analyte to a voltage at the polysilicon gate of the ISFET according to the Nernst equation. This voltage is detectable as a gate threshold shift in the MOSFET. A reference electrode with a fixed potential is required. Without 
further processing, the device can be used for $\mathrm{pH}$ sensing with the native passivation of the CMOS process, $\mathrm{Si}_{3} \mathrm{~N}_{4}$, which is known to show a sub-Nernstian response. Typical responses are $46 \mathrm{mV} / \mathrm{pH}$ [30]. Alternatively, the device may be coated with another dielectric, such as $\mathrm{Ta}_{2} \mathrm{O}_{5}$, which has been shown to give a response as large as $58 \mathrm{mV} / \mathrm{pH}[21]$. $\mathrm{The} \mathrm{Ta}_{2} \mathrm{O}_{5}$ is a thin insulator and therefore does not modify the cross-talk properties of the individual sensors that have previously been shown to be capable of forming images [16].

Using a photomask and photolithography to open a window over the active area of the sensor array, a $100 \mathrm{~nm} \mathrm{Ta}{ }_{2} \mathrm{O}_{5}$ layer was deposited on top of the array passivation using a radio frequency sputtering tool (Plassys, France). We chose to use a $100 \mathrm{~nm}$ thick $\mathrm{Ta}_{2} \mathrm{O}_{5}$ film because it was found by experimentation to give consistent coverage over the topographically varying surface. The resist was then stripped, leaving the $\mathrm{Ta}_{2} \mathrm{O}_{5}$ over the array only, using the lift-off technique. A ceramic $\mathrm{Ta}_{2} \mathrm{O}_{5}$ target was used for sputter coating with a RF power of $300 \mathrm{~W}$, and a gas mixture ratio $\left(\mathrm{Ar}^{\mathrm{O}} \mathrm{O}_{2}\right)$ of 9:1 at room temperature. The working pressure was $100 \mu$ bar. It has been shown that an optimised process can deposit films with an ideal surface stoichiometric ratio and good surface roughness [31]-[33]. As shown in Fig. 4, the sputtering conditions that were used in this work yielded a $\mathrm{Ta}_{2} \mathrm{O}_{5}$ film with an average surface roughness of $0.42 \mathrm{~nm}$ and surface chemical atomic ratio (Ta:O) of 1:1.76. The surface roughness is comparable to earlier work. However, the surface chemical atomic ratio is lower than the ideal stoichiometric value of $1: 2.5$ [31]. A high temperature and pure oxygen atmosphere is incompatible with both the fabrication technique and CMOS technology. As a compromise, the conditions we used allow some oxygen to leech out of the surface during sputtering, affecting the surface stoichiometric ratio. Nevertheless, as will be described, we obtained a significant improvement in device performance.

\section{Chip Packaging}

Prior to performing aqueous measurements, an encapsulation sealant was required to protect the bond pads and wire bonds. Fig. 5 shows the packaging and encapsulation technique used to achieve this protection while leaving the sensor active area exposed. The chip was mounted onto a PGA-144 chip carrier by applying EPO-TEK H74 epoxy to the back-side of the die. The epoxy was then cured at $150{ }^{\circ} \mathrm{C}$ for 10 minutes, bonding the chip to the chip carrier. The chip pads were then wire bonded to the chip carrier. Next, a $3 \mathrm{~mm}$ square Polydimethylsiloxane (PDMS) cube was placed on top of the sensor array (thus protecting the sensor area from the epoxy) and EPO-TEK 302-3M epoxy was used to encapsulate the bond pads and wire bonds. A PDMS cube was used for protection because of its ability to adhere to the surface without any adhesive and the ease of removal. The epoxy was cured at room temperature for 24 hours. The long room temperature cure time eliminates the risk of the epoxy, the viscosity of which reduces at higher temperature, leaking underneath the PDMS cube. The PDMS cube was then peeled off to create a cavity over the sensor array. A polypropylene centrifuge tube from Fisherbrand was cut and fitted on top of the chip carrier using epoxy to contain the necessary experimental fluids. This packaging technique has proven to be very robust. It is easily repeated from chip-to-chip and once a chip has been packaged, a great many enzyme assays can be performed on the same chip without damage to the underlying electronics.

\section{Instrumentation and Measurement System}

To operate the sensor array chip, a high-speed instrumentation and measurement system was constructed to acquire the pixel voltages (representing the local $\mathrm{pH}$ value) and to provide the digital address signals to the chip. For enzyme kinetics measurements, the speed of the measurement tool was designed to operate up to a maximum speed of $2 \mu \mathrm{s} /$ pixel. This maximum acquisition rate translates to 2.048 milliseconds per frame, which equates to just under 500 frames per second, although this ability is not fully used in this paper

The instrumentation and measurement system consists of a computer, four PXI-Express-6358 X series acquisition cards in a PXI-Express-1073 chassis (National Instruments, UK), a custom printed circuit board (Newbury Electronics, UK) and a packaged chip. An external voltage source was connected to a $\mathrm{Ag} / \mathrm{AgCl}$ quasi-reference electrode [34] which was dipped into the centrifuge tube on top of the chip containing an aqueous solution. The $\mathrm{Ag} / \mathrm{AgCl}$ quasi-reference electrode was formed by immersing $\mathrm{Ag}$ wire in $0.1 \mathrm{M} \mathrm{KCl}$ and then biasing it at $1.3 \mathrm{~V}$ using a potentiostat for 2 minutes. The reference electrode was used in the aqueous solution to set the floating gate potential of all the ISFET sensors in the array.

The PXI-Express chassis was connected to a computer with a PCI Express-8361 card. A PGA ZIF $15 \times 15$ socket was used to house the chip carrier in the PCB. The PCB connects all 64 analog outputs from the chip to the 16 analog inputs of each acquisition card. In addition, the PCB also connects 10 digital outputs from one of the cards to all the respective address inputs on the chip. All the digital outputs from the card are in the range of $0-5 \mathrm{~V}$. Two MAX $3001 \mathrm{E}$ voltage level shifters were used to lower the voltage to a range of $0-3.3 \mathrm{~V}$ to be compatible with the power supply of the chip. Besides connecting all the analog outputs and digital inputs, the PCB also connects a $1 \mathrm{~V}$ voltage source to the gate of every in-pixel load transistor (P1 in Fig. 1a) of the chip.

The whole operation of the system was controlled by the software package LabVIEW that was programmed to read 64 analog outputs from the chip and also send the 10-bit of digital addresses to activate the chip. It is very important to synchronise the digital addressing and the analog data, in order to obtain the correct pixel measurement as a function of time. This was done with the PXI-Express reference clock where both the digital addresses and analog data (at half the period of sampling rate) were synchronised.

For a high speed data storage system, the direct memory access (DMA) feature of the PXI-Express-6358 X series 
acquisition cards and solid state drive (SSD) of the PC was used to send digital addresses and receive analog data respectively. Using this strategy, the system operated independently of the central processing unit and operating system. It was therefore possible to offer continuous addressing without interruptions and for large volumes of data to be transferred to the SSD at $60 \mathrm{MB} / \mathrm{s}$ as a National Instruments standard TDMS file.

For real time analysis and measurement, LabVIEW was used to arrange all the data into frames of 65536 pixels. The data was then used for $2 \mathrm{D}$ and $3 \mathrm{D}$ image representation, as well as data analysis of individual pixels.

\section{E. Chemicals}

For the hexokinase assays, the following reagents were used. Hexokinase and glucose-6-phosphate dehydrogenase (G6PDH), both purified from Saccharomyces cerevisiae, were purchased from Sigma-Aldrich. Adenosine 5'-triphosphate (ATP) disodium salt and triethanolamine hydrochloride were also sourced from Sigma-Aldrich. $\beta$-Nicotinamide adenine dinucleotide phosphate ( $\beta$-NADP) was purchased from Applichem, D-glucose from Fisher Scientific, and magnesium chloride hexahydrate from VWR.

Phosphate buffer at $\mathrm{pH} 7$ was purchased from SigmaAldrich for washing steps. Solutions of defined $\mathrm{pH}(\mathrm{pH} 1-$ 14) were made with hydrochloric acid and sodium hydroxide. Buffered $\mathrm{pH} 4$ and $\mathrm{pH} 10$ solutions were bought from Fisher Scientific to characterise the chip sensitivity.

\section{RESULTS AND DISCUSSION}

\section{A. $p H$ Sensitivity, Range and Drift}

The functionality and $\mathrm{pH}$ response of the ISFET sensors were initially tested to demonstrate the ability to detect hydrogen ions using different $\mathrm{pH}$ solutions ranging from $\mathrm{pH} 1$ - 14, prepared from $1 \mathrm{M}$ hydrochloric acid $(\mathrm{pH} 0)$ and $1 \mathrm{M}$ sodium hydroxide ( $\mathrm{pH}$ 14). Experiments were conducted by adding first either the alkali or the acid to the chip and then modifying the $\mathrm{pH}$ by titrating the acid or alkali accordingly, on to the chip. At each titration point the $\mathrm{pH}$ solution was measured for 2 minutes to obtain a stable result. Deionised water was used to clean and rinse the sensor between each $\mathrm{pH}$ measurement. Measurements were taken two times with descending $\mathrm{pH}$ and once with increasing $\mathrm{pH}$ using the same chip to validate that the sensor was able to detect a wide $\mathrm{pH}$ range with good uniformity across the sensor array. The data is shown in Fig. 6. The $\mathrm{pH}$ sensitivity is approximately $45 \mathrm{mV} / \mathrm{pH}$ and yields a near linear voltage response between $\mathrm{pH} 2-12$. We noted that this is less than the potential $58 \mathrm{mV} / \mathrm{pH}$ for $\mathrm{Ta}_{2} \mathrm{O}_{5}$. The signal loss may be due to the nonstoichiometric nature of the $\mathrm{Ta}_{2} \mathrm{O}_{5}$ we deposited or attenuation in the voltage-follower circuit of each pixel.

Besides sensitivity and a wide working $\mathrm{pH}$ range, the $\mathrm{Ta}_{2} \mathrm{O}_{5}$ sensing layer has an additional advantage: the constant signal drift of $\mathrm{Ta}_{2} \mathrm{O}_{5}$ ISFET is approximately only $0.8 \mathrm{mV} /$ hour, as shown in Fig. 7, as compared to $10 \mathrm{mV} /$ hour [17] for the $\mathrm{Si}_{3} \mathrm{~N}_{4}$ passivation layer of the chip when used as a $\mathrm{pH}$ sensor. Buffered $\mathrm{pH} 7$ solutions were used for these drift measurements. The solution was added and measurements were taken for up to 2.5 hours. The measurement of the drift was taken from the average of the whole sensor array. This low drift value allows this sensor to be used for stable assay measurements, unlike other ISFET devices which can suffer from significant levels of drift [17], [30], since our measurements only require 15 minutes to acquire sufficient enzyme response data. The chip is also very robust and each sensor can be reused several times.

\section{B. Signal Noise and Number of Pixels}

Our measurements produce a complete independent data set for all pixels. Using the array we can take data from a single pixel, or we can average data from 2 to $2^{16}$ pixels. The effect of averaging is to reduce the mean square (MS) noise of the combined assay signal. To illustrate, in Fig. 8 we show a typical time-course from the chip for a small concentration of glucose. In this example, it can be seen for the data taken from a single pixel, the noise is very large. However, if we plot the data obtained by averaging the signal from $2^{16}$ pixels in the array, then the noise is much reduced. We have analysed this characteristic of the system in detail.

For any single pixel in the array, the voltage signal, including its noise component is $V_{i}$. The MS voltage noise is $e_{i}^{2}$. For a data sample in the interval from time $t_{1}$ to $t_{2}, e_{i}^{2}$ is calculate using,

$$
e_{i}^{2}=\frac{1}{t_{2}-t_{1}} \int_{t_{1}}^{t_{2}} V_{i}^{2}(t) \mathrm{d} t
$$

The interval $t_{1}$ to $t_{2}$ is chosen to be a region of data where the signal is not changing. When the signal is averaged for $M$ independent pixels, the average signal is calculated to be,

$$
V_{M}=\frac{1}{M} \sum_{i=1}^{M} V_{i}(t)
$$

We can therefore calculate the noise for the average voltage $V_{M}(t)$ using (2). The results of this analysis are presented in Fig. 9, where we plot the MS noise as a function of $\frac{1}{\sqrt{M}}$. As can be seen the graph is very nearly linear, which is what we expect from Gaussian statistics.

Using this data, we are able to make practical decisions about the number of pixels required to make usable measurements for the assay conditions we have chosen. For the substrate glucose concentration we described, chosen to be the physiological range for in human blood. We found that 5 pixels provide adequate data.

Using the array chip, we can program the number of pixels used and therefore target different substrate concentrations. Table I shows the physiological range of glucose in different body fluids. Whilst standard practice today is to measure 
blood glucose, a more sensitive system may be able to perform the assay reliably in other fluids such as urine or tears. However, glucose is not a functional substrate in either urine or tears, so further clinical study would be required to determine the efficacy of such an approach.

TABLE I

PHYSIOLOGICAL GLUCOSE LEVELS IN DIFFERENT BODY FLUIDS

\begin{tabular}{|l|c|c|c|}
\hline \multirow{2}{*}{ Body Fluid } & \multicolumn{2}{|c|}{ Glucose Levels (mM) } & \multirow{2}{*}{ References } \\
\cline { 2 - 4 } & Normal & Diabetic & \\
\hline Blood & $3.89-5.56$ & 7 and higher & {$[35],[36]$} \\
\hline Tears & $0.06-0.34$ & $0.4-1.44$ & {$[36]$} \\
\hline Saliva & $0.33-0.35$ & $0.59-0.63$ and higher & {$[37]$} \\
\hline Urine & $0.1-0.5$ & $2.78-5.55$ & {$[38]$} \\
\hline Sweat & $0.28-1.11$ & - & {$[39],[40]$} \\
\hline
\end{tabular}

\section{Spectrophotometric Measurement of Hexokinase Activity}

The enzymatic activity of hexokinase evaluated using a coupled reaction with G6PDH is the reference method for measuring glucose by spectrophotometry. The reactions are shown in (4).

D-Glucose + ATP $\stackrel{\text { Hexokinase }}{\longrightarrow}$ D-Glucose-6-P+ADP $+\mathrm{H}^{+}$
D-Glucose-6-P+ $\beta$-NADP $\stackrel{\text { G6PDH }}{\longrightarrow} 6-\mathrm{PG}+\beta-\mathrm{NADPH}+\mathrm{H}^{+}$

where D-Glucose-6-P is D-Glucose-6-Phosphate, ADP is Adenosine 5'-Diphosphate, 6-PG is 6-Phospho-D-Gluconate and $\beta$-NADP is $\beta$-Nicotinamide adenine dinucleotide phosphate.

Prior to commencing experimental studies using the ISFET array, laboratory verification of hexokinase activity was carried out using a classical assay by monitoring $\beta-\mathrm{NADPH}$ production using a UV-2550 Spectrophotometer (Shimadzu, Japan) at $340 \mathrm{~nm}$ and $25^{\circ} \mathrm{C}$. The concentrations of each reagent in $1.26 \mathrm{ml}$ solution were as follows: $20 \mathrm{mM}$ triethanolamine buffer $\mathrm{pH} 8.5,219 \mathrm{mM}$ glucose, $7.9 \mathrm{mM}$ $\mathrm{MgCl}_{2}, 1.1 \mathrm{mM} \beta$-NADP, $0.7 \mathrm{mM}$ ATP, 1.25 Units G6PDH and $0.02-5$ Units hexokinase. A Unit of hexokinase activity is defined to be the amount of hexokinase required to phosphorylate $1 \mu \mathrm{mol}$ of glucose per minute under standard conditions. Different Units of hexokinase were titrated to determine the optimal amount to use for subsequent experiments. The progress curves that were generated gave suitable kinetics at between 0.125 and 5 Units on the spectrophotometer.

The Michaelis-Menten constant was determined by incubating 2.5 Units of hexokinase with glucose concentrations in the range of $0.2 \mathrm{mM}-22 \mathrm{mM}$. Each progress curve shows a rapidly rising (linear) signal before reaching saturation. From the progress curves, the initial velocity (covering the linear part of the progress curve for each substrate concentration) was plotted versus the substrate concentration and GraphPad Prism, which has a built-in Michaelis-Menten algorithm, was used to calculate the value of $K_{M}$, which was $0.85 \mathrm{mM}$. The data is shown in Fig. 10 .

\section{Enzyme Kinetics Measurement on Chip}

For the on-chip assay, the reagents used for the above spectrophotometer evaluation were simplified, since there is no need to couple the reaction with G6PDH so as to detect the production of $\beta$-NADPH. The second reaction in (4) could therefore be eliminated. Instead the production of protons is measured directly as a $\mathrm{pH}$ change using an ISFET.

Routinely, enzyme assays employ a buffer system to sequester fluxes in protons in order to maintain an optimum $\mathrm{pH}$ range for the enzyme. Thus, the concentration of the buffer plays a crucial role towards the sensitivity of an assay that measures a change in $\mathrm{pH}$. Moreover, it is also essential that reaction components do not alter the $\mathrm{pH}$ intrinsically prior to initiation of the enzyme reaction. Therefore, before testing on the ISFET, reaction conditions were rigorously tested using a S220 SevenCompact ${ }^{\mathrm{TM}} \mathrm{pH} /$ Ion meter (Mettler-Toledo, USA) in order to optimise conditions and maximise sensitivity. A lowering of $\mathrm{pH}$ was evident when all hexokinase reaction components were added to the assay under optimised conditions (data not shown).

The ISFET sensor detects differences in local $\mathrm{pH}$ caused during an enzyme reaction allowing determination of the Michaelis-Menten constant. To maximise the sensitivity of the measurement of enzyme activity on an ISFET sensor, the triethanolamine buffer concentration was reduced from $22 \mathrm{mM}$ to $2.2 \mathrm{mM}$, which was sufficient to maintain the $\mathrm{pH}$ of the solution within the optimum $\mathrm{pH}$ range of the hexokinase $(\mathrm{pH} 7.5$ - 9.0), allowing for a detectable increase in the voltage of the ISFET sensor.

The starting $\mathrm{pH}$ of the buffer was chosen to be 8.5 ; this is at the high end of the desired operating range for the enzyme, since the $\mathrm{pH}$ falls in the course of the reaction. The $\mathrm{pH}$ of the ATP solution was adjusted from $\mathrm{pH} 3.5$ to $\mathrm{pH} 8.5$ by titrating with $1 \mathrm{M}$ sodium hydroxide thus preventing an intrinsic reduction of $\mathrm{pH}$ below the enzyme's working range.

The data for all experiments present in this paper was acquired from the same chip to ensure consistency. The chip was cleaned thoroughly with phosphate buffer solution and blown dry with a high pressure nitrogen gun between each experiment. We also demonstrated to our satisfaction that two more chips performed to the same standard and function. For the three chips we measured, there was a slight chip-to-chip variation in performance of approximately $1 \mathrm{mV}$ in the signal voltage when measuring the response to the addition of a $0.7 \mathrm{mM}$ substrate. Complete datasets were obtained from each of the chips. For the data acquired in Fig. 11, two $1 \mathrm{ml}$ solutions were prepared with reagent concentrations as follows: (solution A) $22 \mathrm{mM}$ triethanolamine buffer $\mathrm{pH} 8.5$, $239 \mathrm{mM}$ glucose, $8.6 \mathrm{mM} \mathrm{MgCl} 2$ and 2 Units hexokinase; (solution B) $2.2 \mathrm{mM}$ triethanolamine buffer $\mathrm{pH} 8.5,239 \mathrm{mM}$ glucose, $8.6 \mathrm{mM} \mathrm{MgCl} 2$ and 2 Units hexokinase. As an aid to checking our work, $43 \mu \mathrm{g} / \mathrm{ml}$ phenol red was added to each solution to provide a visual $\mathrm{pH}$ indicator. Experiments were then conducted by varying the concentration and $\mathrm{pH}$ of ATP and testing for hexokinase activity. Additional control experiments were carried out whereby hexokinase was replaced with bovine serum albumin (BSA) as a nonenzymatic protein control. Fig. 11a shows the results obtained 
when the buffer concentration was $22 \mathrm{mM}$ and Fig. $11 \mathrm{~b}$ shows the results when the buffer concentration was reduced to 2.2 $\mathrm{mM}$.

As can be seen, the response to changing conditions of ATP and $\mathrm{pH}$ is greater with $2.2 \mathrm{mM}$ buffer, showing proton detection is higher at the lower buffer concentration. Additionally, there is little or no positive response when BSA was used in place of hexokinase. With the reaction conditions optimised, an experiment was carried out to test the sensitivity to varying concentrations of glucose in the known physiological range. By doing so, the $K_{M}$ of hexokinase on the ISFET chip could be derived. The range of glucose concentrations tested (between $0.05 \mathrm{mM}-231 \mathrm{mM}$ ) encompassed the physiological range of blood glucose, from $2.8 \mathrm{mM}$ (hypoglycaemia) to $20 \mathrm{mM}$ (extreme hyperglycaemia) [41]-[44]. As can be seen in Fig. 12a, the signal from a typical single pixel (pixel $X$ ) rises towards a fixed voltage plateau that varies as a function of the glucose concentration in the analyte. Fig. 12b shows the average signal as a function of time for $N=5$ pixels selected from the chip. This procedure reduces the voltage signal noise by $\sqrt{N}$ as would be expected to be $1.1 \mathrm{mV}$, and enables the calculation of a standard deviation. At the voltage plateau the rate of turnover of glucose by the enzyme is constant for the given concentration. Since the turnover of glucose is directly related to the production of protons, and the ISFET gives a voltage signal that is linear with $\mathrm{pH}$, we have plotted the average maximum (plateau) signal voltage against log [glucose] for pixel $X$ in Fig. 12c. Fig. $12 \mathrm{~d}$ shows the same plot for the average of five pixels. We observe that the $\log / \mathrm{lin}$ plot has a straight-line dependency, as we expect, through a central operating region that, for the assay conditions we have used, is appropriate for the physiological range of glucose in blood. The plot deviates from the Nernstian fit at both the low and very high glucose concentrations. The assay is not sufficiently sensitive for the very lowest concentrations for the assay chemistry used, whereas at very high glucose concentrations, the ATP is completely used up by the glucose, saturating the signal.

In Fig. 12e and $12 \mathrm{f}$ we show Lineweaver-Burk double reciprocal plots of $1 /(\max (\mathrm{d} V / \mathrm{d} t))$ vs $1 /$ [glucose] for each concentration that was used. As expected, these plots show a linear region, the intercept with the $1 /$ [glucose] axis of which can be used to calculate $1 / K_{M}$, by rearranging (1) as shown in (5).

$$
\frac{1}{v}=\frac{K_{M}}{v_{\max }} \frac{1}{[S]}+\frac{1}{v_{\max }}
$$

As with the $\log / \operatorname{lin}$ plots, the assay deviates from linear behaviour for very low or very high [glucose]. Using this method a $K_{M}$ value of $0.58 \pm 0.36 \mathrm{mM}$ for the average of the 5 pixels was obtained. The result is in good agreement with the data that we obtained for the same assay using the spectrophotometer.

\section{CONCLUSION}

In this paper, we have demonstrated an enzyme assay performed using CMOS ISFETs. Using the ISFETs, a signal voltage was produced that tracked the evolution of hydrogen ions in the hexokinase/glucose assay that we used. The data we obtain shows for the first time that enzyme kinetics, and the determination of the Michaelis-Menten constant can be carried out on a CMOS chip. The results are comparable to those obtained using a standard bench-top spectrophotometerbased technique.

In order to make the study of enzyme kinetics possible on a CMOS chip we have developed two new techniques. Using the ISFET array method, we were able to exploit the statistics of averaging signals from more than one channel to reduce the overall noise in the system. Improvements in the assay chemistry were also introduced. By adjusting the buffering of the reagents we have shown that we can increase the local variation in $\mathrm{pH}$ at the ISFET sensors. Using both these techniques, glucose concentrations in the range of $0.05 \mathrm{mM}-$ $231 \mathrm{mM}$ were detected, encompassing the natural physiological range of glucose in blood, from $2.8 \mathrm{mM}$ (hypoglycaemia) to $20 \mathrm{mM}$ (extreme hyperglycaemia). The Michaelis-Menten constant was calculated to be $0.58 \pm 0.36 \mathrm{mM}$. The ISFET data we obtained is in good agreement with traditional spectrophotometer data, which is presented in this paper.

For the hexokinase assay we performed, we found that using five sensors was sufficient. The ISFET device described here has a large array of individually addressable sensors. Since as few as five sensors is adequate for one assay, we anticipate that, by using more area of the array, sensors can be grouped into zones on the surface of the chip. Using this method, in tandem with microfluidics, different zones could be functionalised with different enzymes to measure several metabolites at the same time. In this way, the array architecture can provide two distinct outcomes: noise reduction by averaging over independent channels; and multiple simultaneous metabolomic assays.

A well-known application for this device could be to monitor the symptoms of diabetes mellitus, a chronic metabolic disorder that is increasing in prevalence to an alarming degree, placing a heavy outlay on social and medical resources [44]. As interest grows in metabolomics for precision medicine [28], [29], the work in this paper presents a pathway towards new and exciting handheld diagnostic tools.

\section{ACKNOWLEDGMENT}

The authors wish to thank Dr Rasmus H. Pederson for the XPS measurement and all the technical staff of the James Watt Nanofabrication Centre at Glasgow University for their contributions. 


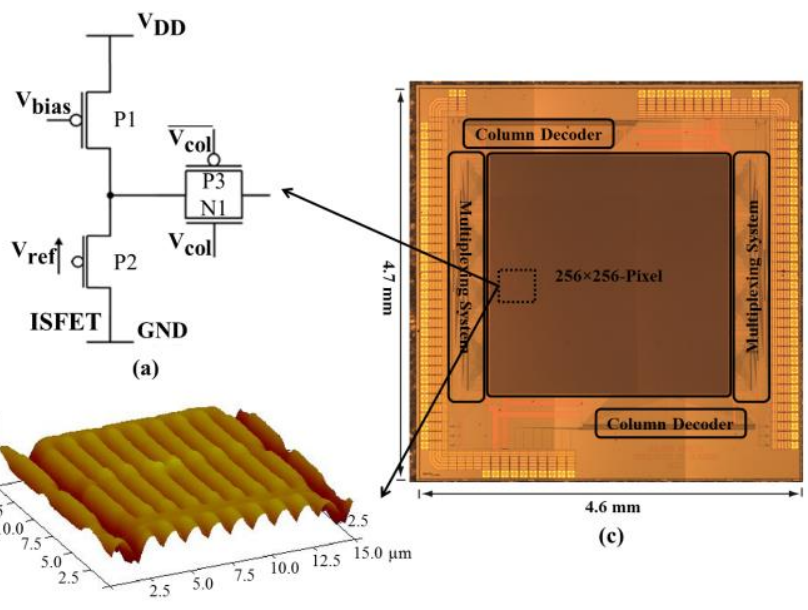

(b)

Fig. 1. (a) A schematic diagram of a single pixel [16] and (b) the surface profile of a single pixel from an atomic force microscope. (c) A microphotograph of the whole of the $256 \times 256$-pixel ISFET sensor array.

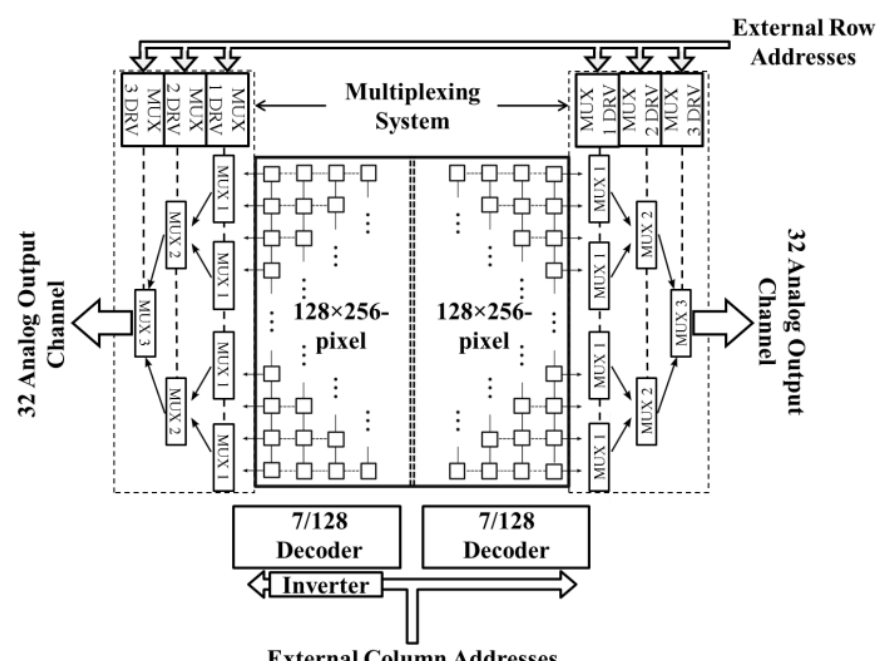

Fig. 2. A schematic diagram of the $256 \times 256$ ISFET sensor array chip, describing the addressing architecture.

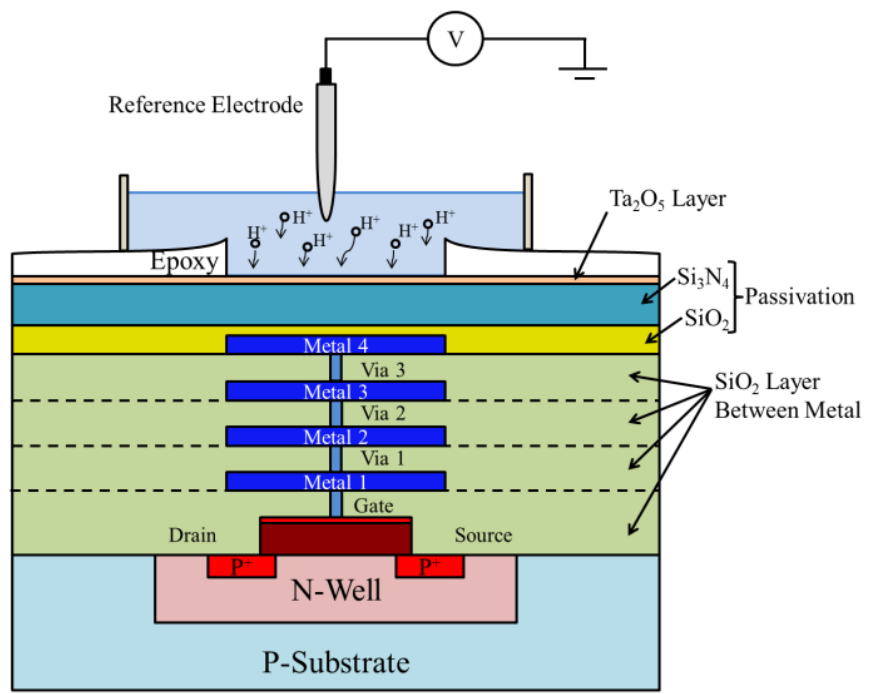

Fig. 3. A cross sectional view of the ISFET structure fabricated using the AMS $0.35 \mu \mathrm{m}$, four metal, process. An additional $\mathrm{Ta}_{2} \mathrm{O}_{5}$ thin film was subsequently added by a post-processing step.

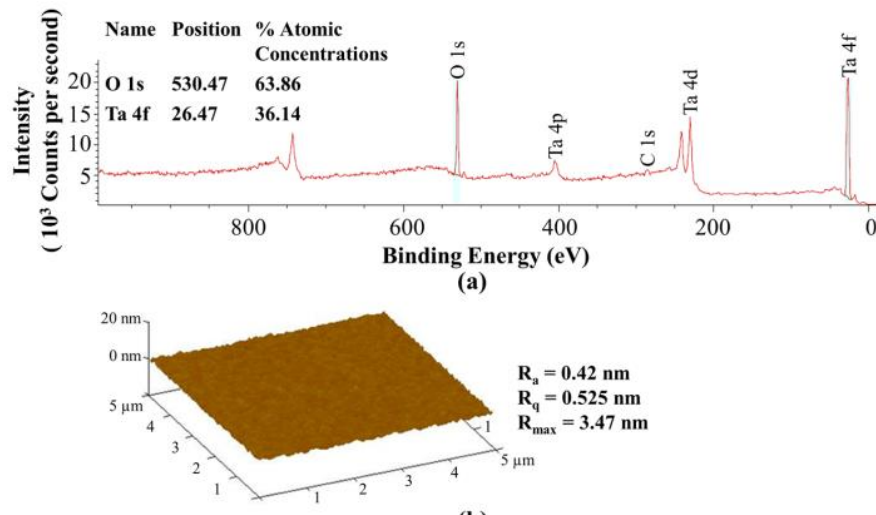

(b)

Fig. 4. (a) X-Ray Photoelectron Spectroscopy (XPS) analysis of the material composition on the $\mathrm{Ta}_{2} \mathrm{O}_{5}$ surface, showing the binding energy and percentage atomic concentrations of $\mathrm{O} 1 \mathrm{~s}$ and $\mathrm{Ta} 4 \mathrm{f}$. (b) The surface profile of the $\mathrm{Ta}_{2} \mathrm{O}_{5}$ layer obtained using Atomic Force Microscopy (AFM). $R_{a}$ is the average roughness, $R_{q}$ is the RMS roughness and $R_{\max }$ is the maximum roughness.

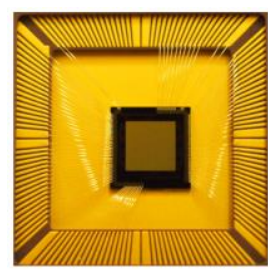

(a)

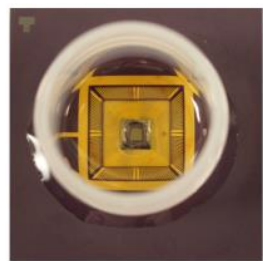

(b)

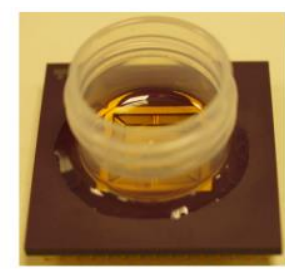

(c)
Fig. 5. Photographs of (a) a fully wire bonded and well encapsulated $256 \times$ 256 pixels ISFET sensor array chip showing (b) top view and (c) side view.

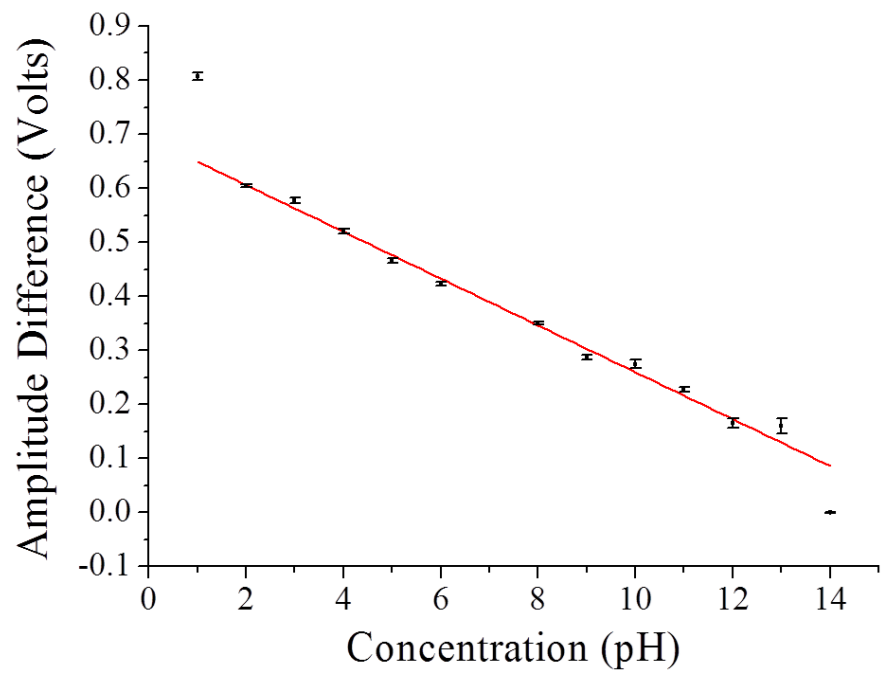

Fig. 6. $\mathrm{pH}$ response curve of the average sensitivity for all the $256 \times 256$ pixels on the chip. The error bars are calculated using the standard deviation. 


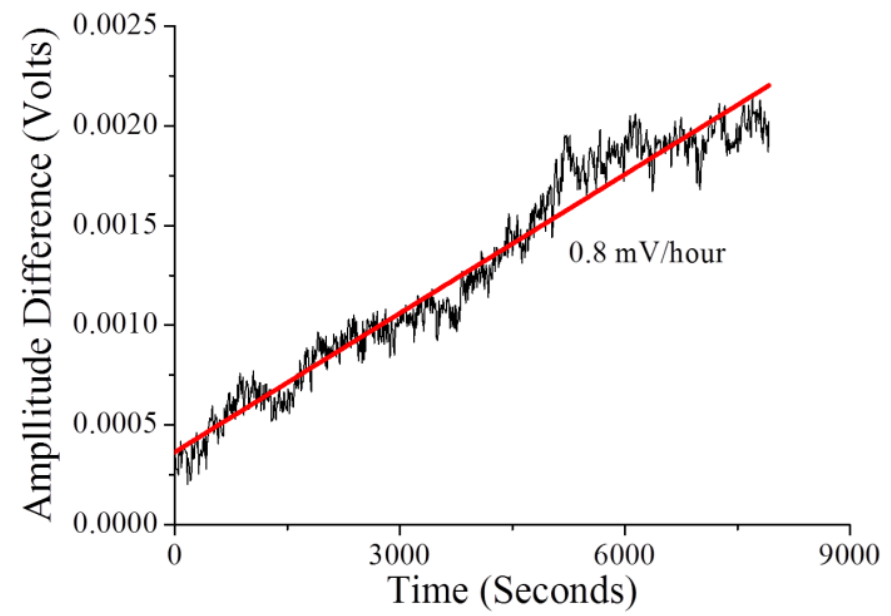

Fig. 7. The average drift of $\mathrm{Ta}_{2} \mathrm{O}_{5}$ sensing layer on the whole array of the CMOS ISFET chip.

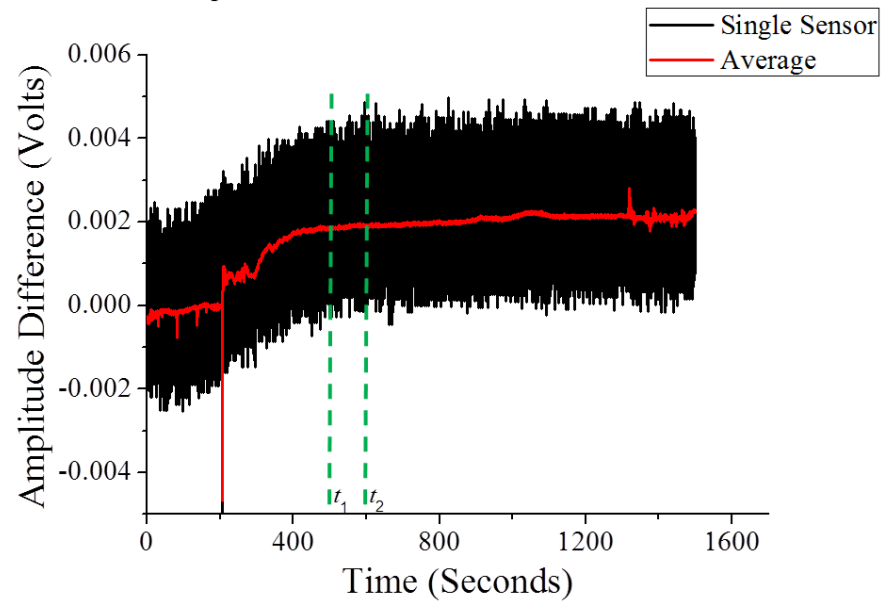

Fig. 8. The comparison of the signal from a typical sensor and the average of the whole array.

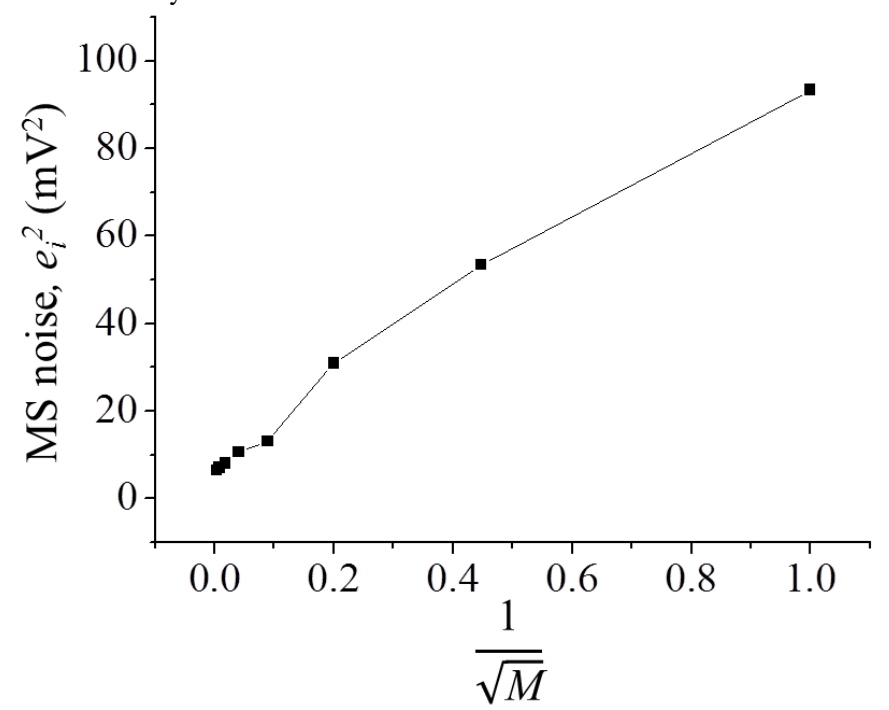

Fig. 9. The MS noise, $e_{i}{ }^{2}$ as a function of $1 / \sqrt{M}$, where $M$ is the number of sensors we choose to average.

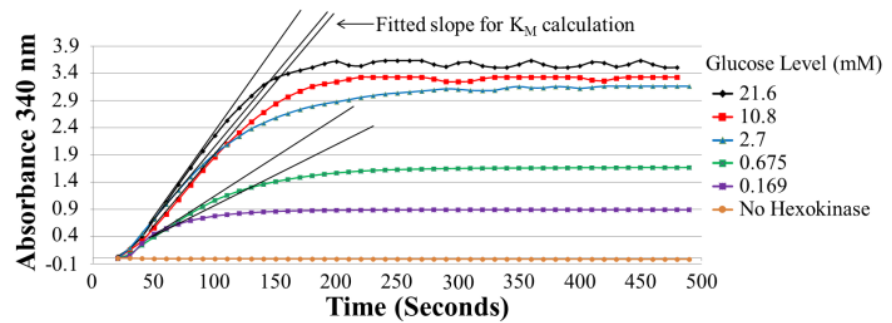

Fig. 10. The data obtained with different glucose concentrations using a spectrophotometer. It shows the linear maximum velocity part of the data that was used for Michaelis-Menten parameter calculation.

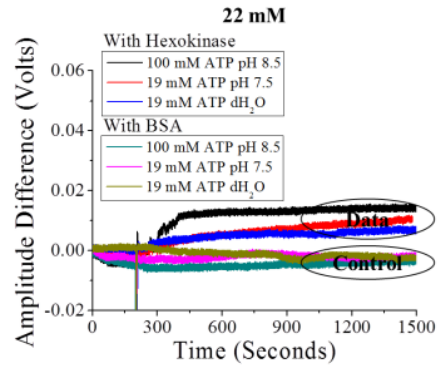

(a)

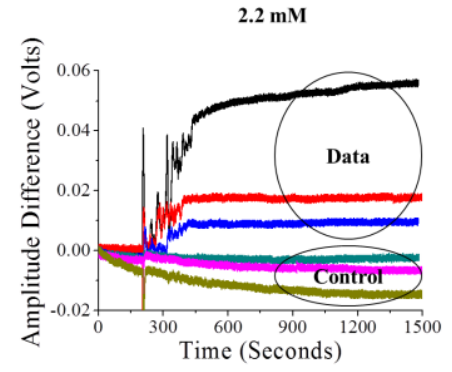

(b)
Fig. 11. On chip enzymatic and control measurements with different concentration and buffer strength of ATP solution in a final buffer concentration of (a) $22 \mathrm{mM}$ and (b) $2.2 \mathrm{mM}$ triethanolamine buffer. The legend applies to both Fig. 9a and Fig. 9b.

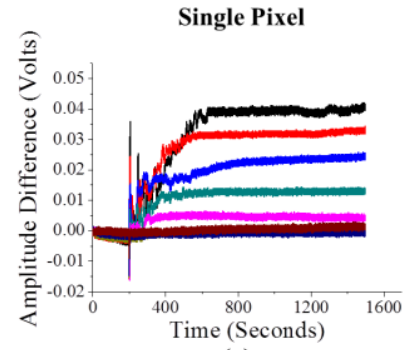

(a)

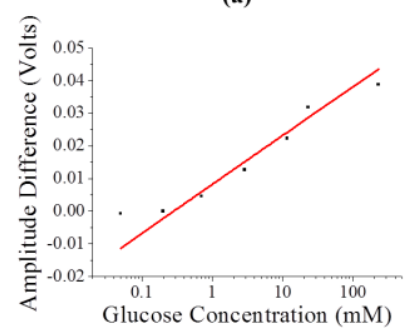

(c)

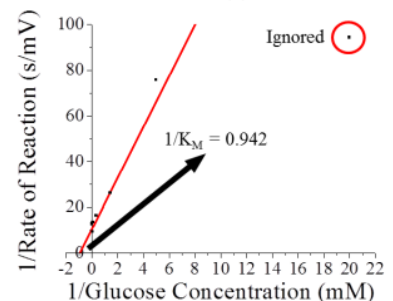

(e)

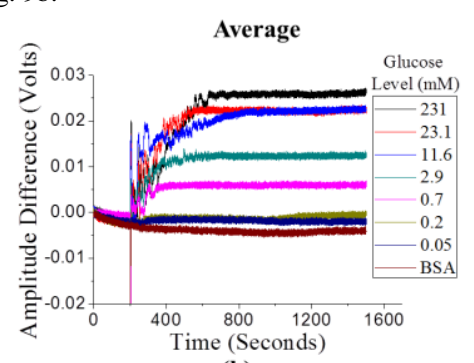

(b)

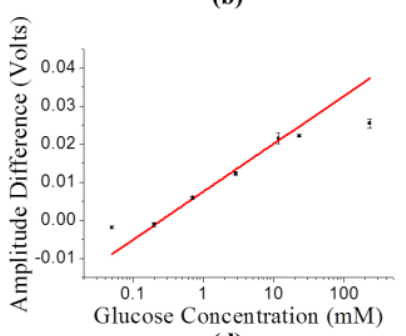

(d)

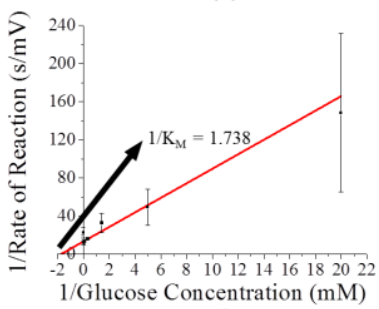

(f)
Fig. 12. (a) A typical single pixel data set acquired from the chip and (b) the average of five pixels. The legend for Fig. 10b is the same as that for Fig. 10a. (c) The voltage change with respect to glucose concentration for a typical single pixel and (d) the average of five pixels. (e) Lineweaver-Burk double reciprocal plot of a typical single pixel and (f) the Lineweaver-Burk plot for an average of five pixels. 


\section{REFERENCES}

[1] R. A. Copeland, Enzymes: A Practical Introduction to Structure, Mechanism, and Data Analysis, 2nd ed., New York, 2000.

[2] R. Chang, Physical Chemistry for the Biosciences, USA, 2005, pp. 363400.

[3] D. L. Purich, Enzyme Kinetics: Catalysis \& Control, 1st ed., USA, 2010.

[4] H. Bisswanger, Enzyme Kinetics: Principle and Methods, Germany, 2002.

[5] C. Pundir, "Introduction to Enzyme and Nanotechnology," in Enzyme Nanoparticles, 2015, pp. 1-7.

[6] E. Seibert and T. S. Tracy, "Fundamentals of enzyme kinetics," Methods in Molecular Biology, vol. 1113, pp. 9-22, 2014.

[7] R. A. Baker and L. Wicker, "Current and potential applications of enzyme infusion in the food industry," Trends in Food Science and Technology, vol. 7, pp. 279-284, 1996.

[8] F. Hasan, A. A. Shah, S. Javed, and A. Hameed, "Enzymes used in detergents: Lipases," African Journal of Biotechnology, vol. 9, pp. 4836-4844, 2010.

[9] E.-H. Yoo and S.-Y. Lee, "Glucose biosensors: An overview of use in clinical practice," Sensors, vol. 10, pp. 4558-4576, 2010.

[10] P. Bergveld, "Development of an ion-sensitive solid-state device for neurophysiological measurements," IEEE Trans. Biomed. Eng., vol. 17, pp. 70-71, 1970.

[11] S. Caras and J. Janata, "Field effect transistor sensitive to penicillin," Analytical Chemistry, vol. 52, pp. 1935-1937, 1980.

[12] C.-S. Lee, S. K. Kim, and M. Kim, "Ion-sensitive field-effect transistor for biological sensing," Sensors, vol. 9, pp. 7111-7131, 2009.

[13] J. Janata, "Historical review. Twenty years of ion-selective field-effect transistors," Analyst, vol. 119, pp. 2275-2278, 1994.

[14] J. Bausells, J. Carrabina, A. Errachid, and A. Merlos, "Ion-sensitive field-effect transistors fabricated in a commercial CMOS technology," Sensors and Actuators B: Chemical, vol. 57, pp. 56-62, 1999.

[15] M. J. Milgrew, M. O. Riehle, and D. R. S. Cumming, "A 16×16 CMOS proton camera array for direct extracellular imaging of hydrogen-ion activity," 2008 IEEE International Solid-State Circuits Conference (ISSCC), pp. 590-638, 2008.

[16] B. Nemeth, M. S. Piechocinski, and D. R. S. Cumming, "Highresolution real-time ion-camera system using a CMOS-based chemical sensor array for proton imaging," Sensors and Actuators B: Chemical, vol. 171-172, pp. 747-752, 2012.

[17] T. Matsuo and M. Esashi, "Methods of isfet fabrication," Sensors and Actuators, vol. 1, pp. 77-96, 1981.

[18] S. Chen, J. G. Bomer, E. T. Carlen, and A. V. D. Berg, " $\mathrm{Al}_{2} \mathrm{O}_{3} /$ silicon nanoISFET with near ideal nernstian response," Nano Letter, vol. 11, pp. 2334-2341, 2011.

[19] H.-K. Liao, J.-C. Chou, W.-Y. Chung, T.-P. Sun, and S.-K. Hsiung, "Study of amorphous tin oxide thin films for ISFET applications," Sensors and Actuators B: Chemical, vol. 50, pp. 104-109, 1998.

[20] D. Sobczyńska and W. Torbicz, " $\mathrm{ZrO}_{2}$ gate $\mathrm{pH}$-sensitive field effect transistor," Sensors and Actuators, vol. 6. pp. 93-105, 1984.

[21] T. Mikolajick, R. Kühnhold, and H. Ryssel, "The $\mathrm{pH}$-sensing properties of tantalum pentoxide films fabricated by metal organic low pressure chemical vapor deposition," Sensors and Actuators B: Chemical, vol. 44, pp. 262-267, 1997

[22] C.-E. Lue, T.-C. Yu, C.-M. Yang, D. G. Pijanowska, and C.-S. Lai, "Optimization of urea-EnFET based on $\mathrm{Ta}_{2} \mathrm{O}_{5}$ layer with post annealing," Sensors, vol. 11, pp. 4562-4571, 2011.

[23] O. A. Boubriak, A. P. Soldatkin, N. F. Starodub, A. K. Sandrovsky, and A. K. El'skaya, "Determination of urea in blood serum by a urease biosensor based on an ion-sensitive field-effect transistor," Sensors and Actuators B: Chemical, vol. 27, pp. 429-431, 1995.

[24] A. P. Soldatkin, J. Montoriol, W. Sant, C. Martelet, and N. JaffrezicRenault, "Creatinine sensitive biosensor based on ISFETs and creatinine deiminase immobilised in BSA membrane," Talanta, vol. 58, pp. 351357, 2002.

[25] B.-K. Sohn, B.-W. Cho, C.-S. Kim, and D.-H. Kwon, "ISFET glucose and sucrose sensors by using platinum electrode and photo-crosslinkable polymers," Sensors and Actuators B: Chemical, vol. 41, pp. 7-11, 1997.

[26] S. F. Clarke and J. R. Foster, "A history of blood glucose meters and their role in self-monitoring of diabetes mellitus," British Journal of Biomedical Science, vol. 69, pp. 83-93, 2012.

[27] R. A. Gatenby and R. J. Gillies, "Why do cancers have high aerobic glycolysis?," Nature Reviews Cancer, vol. 4, pp. 891-899, 2004.
[28] S. E. Jackson and J. D. Chester, "Personalised cancer medicine", International Journal of Cancer, vol. 137, pp. 262-266, 2015.

[29] V. Mukesh, "Personalized medicine and cancer," Journal of Personalized medicine, vol. 2, pp. 1-14, 2015.

[30] B. D. Liu, Y. K. Su, and S. C. Chen, "Ion-sensitive field-effect transistor with silicon nitride gate for $\mathrm{pH}$ sensing," International Journal of Electronics, vol. 67, pp. 59-63, 1989.

[31] S.-J. J. Wu, B. Houng, and B.-S. Huang, "Effect of growth and annealing temperatures on crystallization of tantalum pentoxide thin film prepared by RF magnetron sputtering method," Journal of Alloys Compounds, vol. 475, pp. 488-493, 2009.

[32] D.-H. Kwon, B.-W. Cho, C.-S. Kim, and B.-K. Sohn, "Effects of heat treatment on $\mathrm{Ta}_{2} \mathrm{O}_{5}$ sensing membrane for low drift and high sensitivity pH-ISFET," Sensors and Actuators B: Chemical, vol. 34, pp. 441-445, 1996.

[33] J. C. Chou and C. N. Hsiao, "Comparison of the pH sensitivity of different surfaces on tantalum pentoxide," Sensors and Actuators B: Chemical, vol. 65, pp. 237-238, 2000.

[34] D. A. Fiedler, M. Koppenol, and A. M. Bond, “An ESR-Electrochemical Cell Which Can Be Used in High and Low Dielectric Solvents Over Wide Ranges of Temperature and Time Domain," Journal of The Electrochemical Society, vol. 142. pp. 862-867, 1995.

[35] American Diabetes Association, "Standards of Medical Care in Diabetes - 2014," Diabetes Care, vol. 37, 2014.

[36] R. P. Agrawal, et al., "Noninvasive Method for Glucose Level Estimation by Saliva," Journal of Diabetes \& Metabolism, vol. 4, pp. 15, 2013.

[37] D. K. Sen and G. S. Sarin, "Tear Glucose Levels in Normal People and in Diabetic Patients," British Journal of Ophthalmology, vol. 64, pp. 693-695, 1980.

[38] L. Su, et al., "Colorimetric Detection of Urine Glucose Based $\mathrm{ZnFe}_{2} \mathrm{O}_{4}$ Magnetic Nanoparticles," Analytical Chemistry, vol. 84, pp. 5753-5758, 2012.

[39] O. Olarte, J. Chilo, J. Pelegri-Sebastia, K. Barbé, and W. V. Moer, "Glucose Detection in Human Sweat using an Electronic Nose," $35^{\text {th }}$ Annual International Conference of the IEEE EMBS, 2013.

[40] J. Moyer, D. Wilson, I. Finkelshtein, B. Wong, and R. Potts, "Correlation between Sweat Glucose and Blood Glucose in Subjects with Diabetes," Diabetes Technology \& Therapeutics, vol. 14, pp. 398402, 2012.

[41] G. Boden, X. Chen, and T. P. Stein, "Gluconeogenesis in moderately and severely hyperglycemic patients with type 2 diabetes mellitus," Am. J. Physiol. Endocrinol. Metab., vol. 280, pp. 23-30, 2001.

[42] R. P. Hoffman, C. A. Sinkey, and E. A. Anderson, "Hypoglycemia increases muscle sympathetic nerve activity in IDDM and control subjects," Diabetes Care, vol. 17, pp. 673-680, 1994.

[43] F. Turturro, E. Friday, and T. Welbourne, "Hyperglycemia regulates thioredoxin-ROS activity through induction of thioredoxin-interacting protein (TXNIP) in metastatic breast cancer-derived cells MDA-MB231," BMC Cancer, vol. 7, pp. 96-102, 2007.

[44] N. Hex, C. Bartlett, D. Wright, M. Taylor, and D. Varley, "Estimating the current and future costs of Type 1 and Type 2 diabetes in the UK, including direct health costs and indirect societal and productivity costs," Diabetes Medicine, vol. 7, pp. 855-862, 2012.

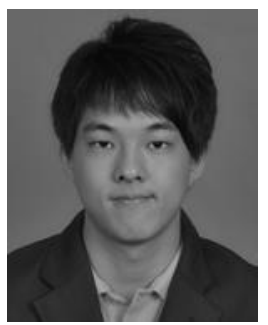

Boon Chong Cheah received the B.Eng. degree in electronic and electrical engineering and M.Sc.(Eng) degree in Nanotechnology and advanced electronic devices from the University of Leeds, Leeds, U.K., in 2010 and 2011 respectively. He is currently pursuing the $\mathrm{Ph} . \mathrm{D}$. degree in electronic and electrical engineering from the University of Glasgow, Glasgow, U.K., where he is with the Microsystem Technology group.

In 2012, he was a Graduate Trainee with the Intel Corporation, Penang, Malaysia. His research interests include microfabrication, bio- and electrochemical sensor, sensor system, and CMOS electronics. 


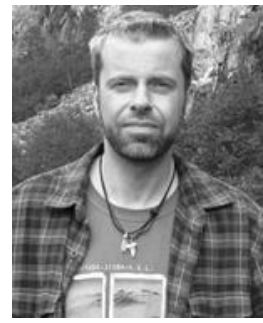

Alasdair Iain MacDonald received a first class B.Sc. (Hons) in molecular biology and was a Wellcome Trust Ph.D. student, both at the University of Glasgow, Glasgow, UK. He then worked in the United States for a period of six years. He has worked in a diverse range of fields including virology, stem cell research, breast and prostate oncology, bacterial transposition and gene regulation in yeast and mammalian cell lines. He is currently employed at the Institute for Infection, Immunity and Inflammation at the University of Glasgow developing enzyme assays on biosensor devices.

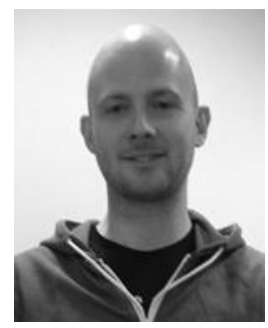

Christopher Martin obtained the MEng degree in Electronics and Electrical Engineering from the University of Glasgow in 2009. He then gained his $\mathrm{Ph} . D$. from the same institution in 2013 in electronics and nanoscale engineering, working as part of the Microsystem Technology Group.

He currently holds the position of post-doctoral research assistant and his interests centre on peripheral nerve repair strategies, wireless and flexible electronics, printing techniques, electrochemistry and bioelectronics.

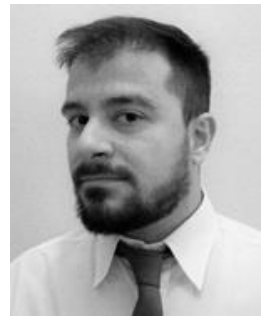

Angelos J. Streklas received his B.Sc. degree in Physics from University of Athens and his M.Sc. degree on Microsystems and Nanostructures from National and Technical University of Athens, Greece. He is currently working towards his Ph.D. degree in Microsystem Technology Group, University of Glasgow, U.K. focusing mainly on CMOS-based ion sensors for chemical and biochemical measurements.

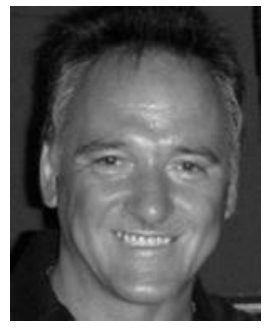

Gordon Campbell has been providing technical research support at Glasgow University since 1972, and has research expertise in Cell Biology, Cell Engineering, Microbiology and Parasitology.https://www.researchgate.net /profile/Gordon_Campbell2?ev=hdr_xprf

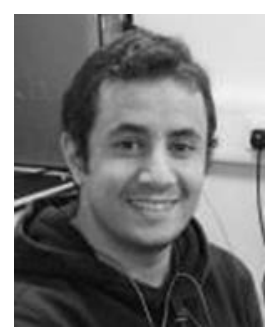

Mohammed A. Al-Rawhani received a B.Sc. in electronics and telecommunication engineering from Amman University, Jordan, in 2004 and an M.Sc. in electrical and electronic engineering from the University of Glasgow, Scotland, UK, in 2007, where he is received his Ph.D in electronic integration design for biomedical applications, in 2012 . His research interests include CMOS low and high-voltage analogue/mixed-signal integrated circuits and front-ends for optical sensing biomedical applications.

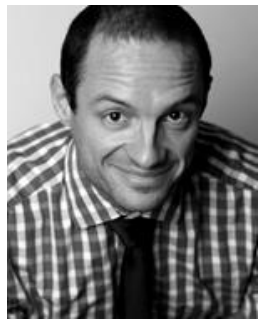

Balazs Nemeth received his M.Sc. degrees in electronic and biomedical engineering from the Budapest University of Technology and Economics, Budapest, Hungary, in 2006 and 2008, respectively. He worked at the Fraunhofer Institute in 2007. He received his Ph.D. degree at the University of Glasgow, Glasgow, U.K. in

2012.

$\mathrm{He}$ is currently with the IMMS GmbH, Erfurt, Germany where he is responsible for the development of bioanalytical and biomedical sensors for applications including point-ofcare and in-vitro diagnostics as well as cancer research.

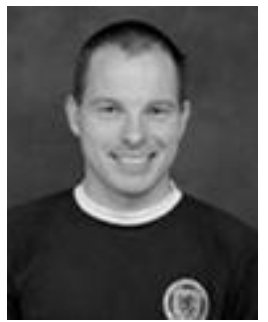

James Grant received the B.Sc. and $\mathrm{Ph} . \mathrm{D}$. in physics from the University of Glasgow, United Kingdom in 2002 and 2006 respectively.

He is currently a Postdoctoral Research Fellow in the School of Engineering at the University of Glasgow where his research interests include nanofabrication, metamaterial devices; plasmonics, terahertz systems, sensors and imaging and CMOS electronics.

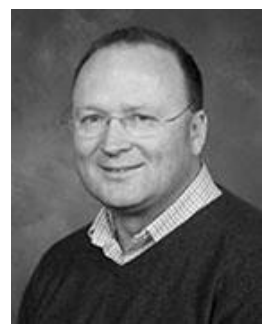

Mike Barrett is Professor of Biochemical Parasitology at Glasgow University and directs the Glasgow Polyomics and the Scottish Metabolomics Facilities. He is deputy Director of the Scottish Universities Life Sciences Alliance (SULSA) and Associate Director of the US based Consortium of Parasitic Drug Development (CPDD). He chaired the Kinetoplastids drug efficacy working group at the World Health Organisation (WHO). He has pioneered application of novel technologies to diagnostics and new anti-microbial interventions and the use of microfluidics for separation of parasites from mammalian body fluids.

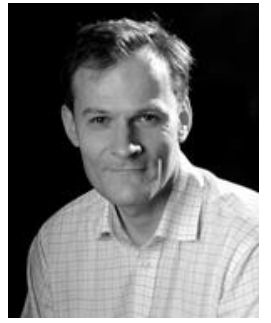

David Cumming (FRSE, FIEEE) holds a B.Eng. in Electronic and Electrical Engineering (Glasgow, 1989) and a PhD in Director of the Electronics Design Centre for Heterogeneous Systems and leads the Microsystem Technology Group in the School of Engineering. He is a cofounder of Glasgow University spin-out Mode Diagnostics, and his research on CMOS ion sensitive array technology has also been commercialized into the Ion Torrent next generation sequencing system. He is a Royal Society Wolfson Merit Award Holder. 